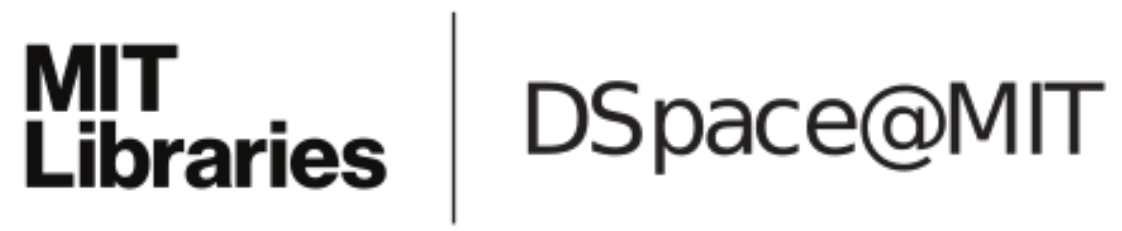

\author{
MIT Open Access Articles
}

\begin{abstract}
Molecular dependencies of dynamic stiffening and strengthening through high strain rate microparticle impact of polyurethane and polyurea elastomers
\end{abstract}

The MIT Faculty has made this article openly available. Please share how this access benefits you. Your story matters.

Citation: Sun, Yuchen et al. "Molecular dependencies of dynamic stiffening and strengthening through high strain rate microparticle impact of polyurethane and polyurea elastomers." Applied Physics Letters 115, 9 (2019): 093701 @ 2019 AIP Publishing

As Published: http://dx.doi.org/10.1063/1.5111964

Publisher: AIP Publishing

Persistent URL: https://hdl.handle.net/1721.1/123521

Version: Author's final manuscript: final author's manuscript post peer review, without publisher's formatting or copy editing

Terms of use: Creative Commons Attribution-Noncommercial-Share Alike 


\title{
Molecular Dependencies of Dynamic Stiffening and Strengthening through High Strain Rate Microparticle Impact of Polyurethane and Polyurea Elastomers
}

\author{
Yuchen $\operatorname{Sun}^{1,2, *}$, You-Chi Mason $\mathrm{Wu}^{1,2}$, David Veysset ${ }^{2}$, Steven E. Kooi ${ }^{2}$, Weiguo $\mathrm{Hu}^{4}$, \\ Timothy M. Swager ${ }^{1,2}$, Keith A. Nelson ${ }^{1,2}$, Alex J. Hsieh ${ }^{2,3}$ \\ ${ }^{1}$ Department of Chemistry, MIT, Cambridge, Massachusetts 02139, USA \\ ${ }^{2}$ Institute for Soldier Nanotechnologies, MIT, Cambridge, Massachusetts 02139, USA \\ ${ }^{3}$ U.S. Army Combat Capabilities Development Command, Army Research Laboratory, \\ FCDD-RLW-MG, Aberdeen Proving Ground, Maryland 21005-5069, USA \\ ${ }^{4}$ University of Massachusetts, Department of Polymer Science \& Engineering, Amherst, MA 01003, USA
}

Citation: Appl. Phys. Lett. 115, 093701 (2019); doi: 10.1063/1.5111964

Online: https://aip.scitation.org/doi/10.1063/1.5111964

Published by the American Institute of Physics

This study investigates the molecular dependencies of dynamic stiffening and strengthening through comparison of high strain rate impact responses of various polyurethanes and polyureas. We use an in-house designed tabletop micro-impact experimental platform-the laser-induced particle impact test (LIPIT) - to perform high strain rate impacts and measure the corresponding material response. Dynamic mechanical analysis and differential scanning calorimetry are used to show that glass transition temperature is a useful predictor of impact response at ambient temperatures. Meanwhile, solid-state nuclear magnetic resonance spectroscopy identifies segmental dynamics as an important determinant of the variation in both dynamic stiffening and strengthening. The impact responses of polyurethanes and polyureas both show clear dependencies on the molecular weight of the soft segment. This comparison suggests the state of intermolecular hydrogen bonding plays a key role in dynamic stiffening and strengthening. This study aims to identify the molecular dependencies of impact response and establish a foundation for further design and testing of optimal high strain rate characteristics in synthetic elastomers.

\footnotetext{
* Corresponding author.

E-mail address: ysun7@ mit.edu (Y. Sun).
} 
Segmented elastomers including polyurethanes ${ }^{1-4}$, polyureas ${ }^{5-12}$, and poly(urethane urea)s ${ }^{13-21}$ are a unique class of chemically versatile materials that have been extensively investigated. These materials possess complex microstructure and span a broad range of physical and mechanical properties. Renewed interest in high-performance segmented elastomers emerged from the experimental realization of their potential for robust ballistic impact response through tailored segmental dynamics ${ }^{5}$. Materials that can exhibit dynamic stiffening, i.e., a shift from rubbery behavior at ambient conditions towards glassy-like behavior upon high strain rate deformation, were shown to be capable of enhanced energy absorption and dissipation ${ }^{5}$. Meanwhile, the influence of molecular moieties has also been reported to be increasingly important on the dynamic behavior of polymers as impulses become faster ${ }^{22}$.

The laser-induced particle impact test (LIPIT) is an in-house designed tabletop method for micro-impact experiments. ${ }^{23}$ The LIPIT is capable of inducing impacts with strain rates on the order of $10^{6}-10^{8} \mathrm{~s}^{-1}$ and has been used for testing high strain rate impact responses in gels ${ }^{24}$, segmented elastomers $^{19-21,25}$, and other various materials ${ }^{26-28}$. Using this platform, Hsieh et al. found that intersegmental mixing between the soft and hard phases strongly affected the high strain rate impact response of poly(urethane urea) and polyurea elastomers ${ }^{21}$. Wu et al. recently used solid-state nuclear magnetic resonance spectroscopy (ssNMR) and attenuated total reflectance-Fourier transform infrared spectroscopy (ATR-FTIR) data along with LIPIT experiments to provide a comprehensive discussion on the importance of an intersegmental hydrogen bonding network for dynamic stiffening in twocomponent polyurethanes without segregated hard domains ${ }^{25}$.

Polyurethanes are ideal candidates for the systematic study of dynamic response due to their widely tunable chemical constituents. Changing particular attributes enables the precise study of various molecular dependencies of dynamic stiffening. As stated above, Wu et al. demonstrated the importance of intersegmental hydrogen bonding by studying two-component polyurethanes with differing isocyanates, either 4,4'-methylene diphenyldiisocyanate (MDI) or hexamethylenediisocyanate (HDI). We further the systematic study of dynamic stiffening with the LIPIT, focusing on synthetic threecomponent polyurethanes based on MDI, butanediol (BDO), and poly(tetramethylene oxide) (PTMO). The addition of the chain extender BDO in these three-component polyurethanes allows greater control over material characteristics including microstructure, hard segment content, and mechanical properties and facilitates study of the effects of chemistry on mechanical behavior. Ultimately, we aim to establish a systematic approach to the design of optimal mechanical properties through chemistry and testing of such synthetic elastomers.

PTMO, MDI, BDO, HDI, and dibutyltin dilaurate were purchased from Sigma-Aldrich and used as received. Tetrahydrofuran (THF) (J.T.Baker) was first dispensed from a solvent purification system (Innovative Technologies) containing activated alumina columns and then stored under argon over $4 \AA$ molecular sieves. A modified-MDI curative, polycarbodiimide-modified diphenylmethane diisocyanate, (Isonate 143L) was purchased from RCS Rocket Motor Components and oligomeric diamines (Versalink® P650 and P1000), based on poly(tetramethylene oxide di-p-aminobenzoate) with the molecular weight of PTMO, 650 and $1000 \mathrm{~g} / \mathrm{mol}$, respectively, from Evonik. Other solvents were purchased from commercial sources and used as received.

The three-component polyurethanes were synthesized using a two-step pre-polymer route. MDI and PTMO were first reacted to form MDI-capped PTMO-MDI oligomers followed by addition of BDO to complete polymerization in a solution phase synthesis to yield MDI-BDO-PTMO based 
polyurethanes. Two polyurethanes were synthesized in this fashion, MDI-BDO-650 and MDI-BDO1000 , where the molecular weight of PTMO is 650 and $1000 \mathrm{~g} / \mathrm{mol}$, corresponding to shorter and longer chain length, respectively. The corresponding weight percentage (wt\%) of hard segment content (\%HS) was calculated using equation 1 shown below as $48 \%$ and $37 \%$, respectively,

$$
\% H S=\frac{(R) M_{d i}+(R-1) M_{b d}}{M_{g}+(R) M_{d i}+(R-1) M_{b d}}
$$

where $R$ is the molar ratio of the diisocyanate to PTMO, and $M_{d i}, M_{b d}$, and $M_{g}$ are the number average molecular weights of MDI, BDO, and PTMO, respectively ${ }^{17}$.

Polyureas were synthesized by mixing a modified-MDI isocyanate curative with an oligomeric diamine in a 1:1 stoichiometric ratio. This synthesis yielded two polyurea polymers, PU-650 and PU1000 , where the molecular weight of PTMO is 650 and $1000 \mathrm{~g} / \mathrm{mol}$, respectively. The weight percent of hard segment was calculated to be $50 \%$ and $33 \%$, respectively.

Micro-impact experiments were conducted with the LIPIT. As illustrated in Fig. 1, the LIPIT uses a high-power laser pulse (Nd-YAG, 532-nm wavelength, 10-ns duration) to eject microparticles from a launching pad towards a target placed $2 \mathrm{~cm}$ below. The launching pad is composed of a glass substrate (210- $\mu \mathrm{m}$ thick), a sacrificial gold layer (60-nm thick), an elastomer film (polyurea, $40-\mu \mathrm{m}$ thick), and particles distributed onto the film. The high-power laser pulse ablates the gold, expands the film, and ejects a particle at high speeds towards the target. The impact velocity is varied by tuning the energy of the high-power laser pulse. The impact event is illuminated with a second laser pulse (640-nm wavelength, 30- $\mu$ s duration) and is subsequently captured through a microscope objective by an ultrahigh-speed camera (SIMX16, Specialised Imaging) consisting of 16 independently triggered ICCD cameras with a minimum exposure time of $5 \mathrm{~ns}$ and variable interframe time.

In this experiment, silica particles with $7.38-\mu \mathrm{m}$ diameter were accelerated to velocities between 50 and $1300 \mathrm{~m} / \mathrm{s}$. Calculation of strain rate as the ratio between strain, estimated as one for characteristic penetration of one particle diameter, and characteristic contact time, impact velocity over particle diameter, gives strain rates on the order of $10^{6}-10^{8} \mathrm{~s}^{-1}$. Figures 2(a) and 2(b) show representative image

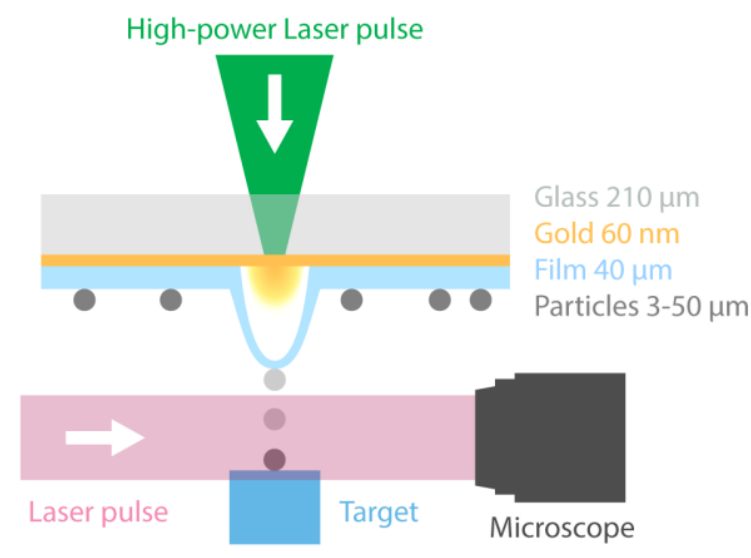

Figure 1. Schematic representation of the LIPIT at the instance when a microparticle is ejected towards a target by laser ablation of a gold layer and subsequent expansion of a film layer. Particle trajectory before and after impact is visualized by a laser pulse and ultra-high-speed camera through a microscope. In this experiment, we access strain rates on the order of $10^{6}-10^{8}$ $\mathrm{s}^{-1}$ using $7.38-\mu \mathrm{m}$ diameter silica particles. 
sequences of impacts against MDI-BDO-650 and MDI-BDO-1000, respectively. Figure 2(a) shows the impact and subsequent rebound of a silica particle against MDI-BDO-650. The trajectories are analyzed to yield impact and rebound velocities of $463 \mathrm{~m} / \mathrm{s}$ and $91 \mathrm{~m} / \mathrm{s}$, respectively. The coefficient of restitution (CoR), defined as the ratio between rebound and impact velocity or equivalently the square root of the ratio between the corresponding kinetic energies, is calculated to be 0.197. Figure 2(b) shows the impact at $510 \mathrm{~m} / \mathrm{s}$ with no rebound evidenced; rather, the particle remains on the surface of MDI-BDO-1000 following impact. In the case of no particle rebound, CoR is zero.

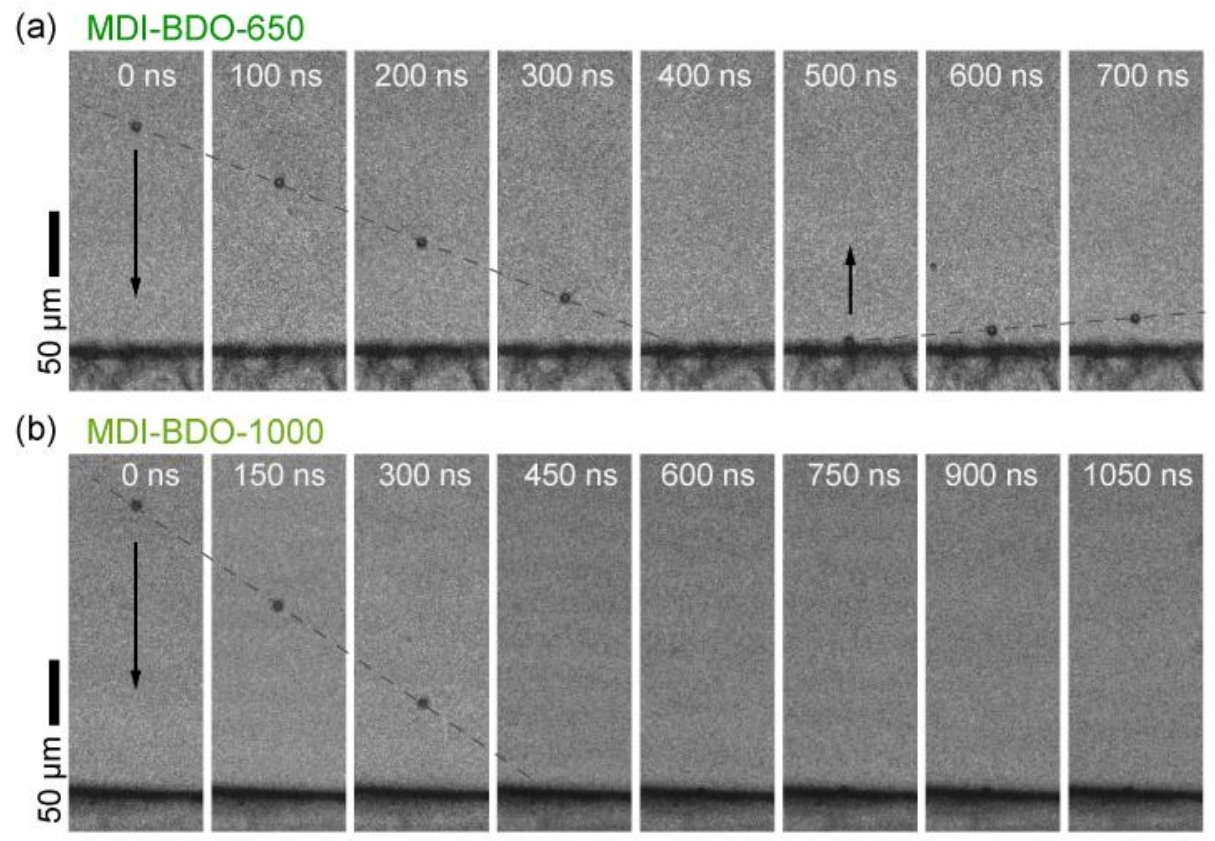

Figure 2. Representative image sequences for (a) impact of a particle on MDI-BDO-650 at 463 $\mathrm{m} / \mathrm{s}$ followed by rebound and (b) impact on MDI-BDO-1000 at $510 \mathrm{~m} / \mathrm{s}$ with no rebound. In both sequences, the particle initially travels downwards towards the target at the bottom of the images. Arrows denote particle direction and dashed lines illustrate particle impact and rebound speeds.

For each material, numerous impacts were performed and analyzed with curves generated to display CoR as a function of impact velocity. These CoR curves act as a comparative measure of dynamic stiffening at the strain rates associated with these high velocity impacts. Further, threshold velocity for a given material is taken as the median of the highest impact velocity inducing rebound and the lowest without rebound. Threshold velocity is reflective of dynamic stiffening and additionally dynamic strengthening and is specific to the material and the impacting particle. In this paper, we use $\mathrm{CoR}$ and threshold velocity as distinct and complementary measures of high strain rate impact response.

As impact velocity and strain rate approaches zero, we expect CoR to trend towards the ideally elastic limit of 1 in all materials. Figure 3 shows the CoR curves for MDI-BDO-650 and other polyurethanes $^{25}$. Clearly at the lowest performed impacts around $50 \mathrm{~m} / \mathrm{s}$, the responses are distinctly inelastic. Notably, MDI-BDO-650 exhibits higher CoR and threshold velocity than MDI-BDO-1000, which demonstrates greater dynamic stiffening and strengthening, respectively. Meanwhile, the CoR of MDI-650 is similar to that of MDI-BDO-1000 at impact speeds up to $500 \mathrm{~m} / \mathrm{s}$, despite the twocomponent MDI-650 having a much lower hard segment content, 28 to $37 \mathrm{wt} \%$. Moreover, MDI-650 has a remarkably higher threshold velocity than MDI-BDO-1000, suggesting a greater sensitivity in CoR 


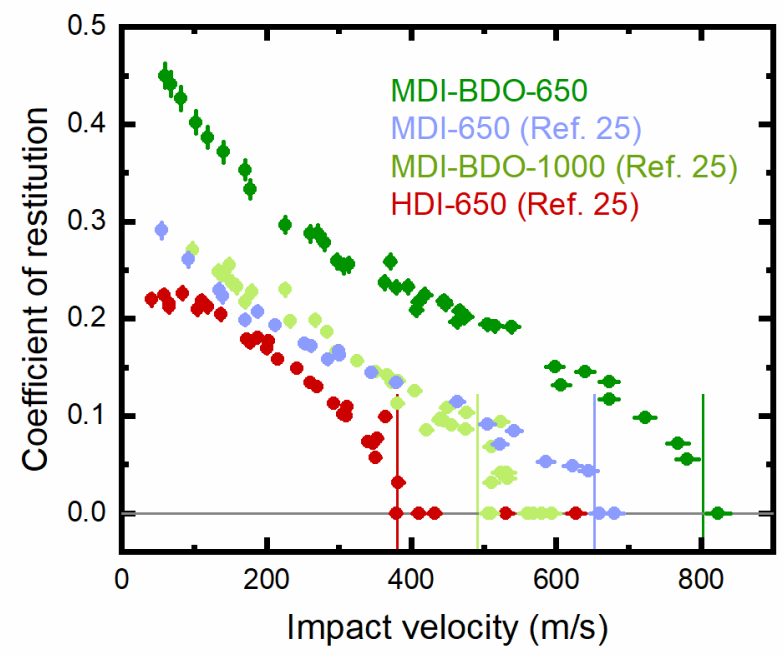

Figure 3. Comparison of coefficient of restitution (CoR) over impact velocity for polyurethanes upon impact by $7.38 \mu \mathrm{m}$ silica spheres. Vertical lines demark threshold velocity for corresponding materials. Error bars are shown for each point. MDI-BDO-650 shows the greatest CoR and threshold velocity which corresponds to more extensive dynamic stiffening and strengthening, respectively. MDI-650 and MDI-BDO-1000 exhibit similar curves, though MDI-650 has a higher threshold velocity. Data for MDI-650, HDI-650, and MDI-BDO-1000 are reproduced from Wu et al. ${ }^{25}$ with permission from Elsevier.

to strain rate. These high strain rate impact response measurements are the foundation on which evaluation of our synthetic elastomers are made.

Glass transition temperatures $\left(\mathrm{T}_{\mathrm{g}}\right)$ were determined for polyurethanes with dynamic mechanical analysis (DMA) at $1 \mathrm{~Hz}$ and differential scanning calorimetry (DSC) at $10^{\circ} \mathrm{C} / \mathrm{min}$. The enthalpy relaxation time for DSC measurements at $10^{\circ} \mathrm{C} / \mathrm{min}$ is often regarded as $100 \mathrm{~s}$, corresponding to an equivalent frequency of $0.0016 \mathrm{~Hz}^{6-8}$. Figure 4 displays these dynamic $\mathrm{T}_{\mathrm{g}}$ values along with the frequency range corresponding to the strain rates probed by LIPIT experiments. An increase in dynamic $\mathrm{T}_{\mathrm{g}}$ of the materials as frequency increases from $0.0016 \mathrm{~Hz}$ in DSC to $1 \mathrm{~Hz}$ in DMA is observed. This trend is indicative of enhanced glassy-like character with increased strain rate. It is notable that the materials shown to undergo glassy transition closest and farthest from room temperature have the highest and lowest CoR, respectively. Further, based on the available data, the low frequency $\mathrm{T}_{\mathrm{g}}$ (from DSC) of MDI-650 is closer to that of MDI-BDO-1000 while its high frequency $\mathrm{T}_{\mathrm{g}}$ (from DMA) shifts toward MDI-BDO-650. This enhanced frequency sensitivity of $T_{g}$ in MDI-650 may result from its amorphous character which promotes intermolecular hydrogen bonding between amide protons and ether carbons, opposed to the semi-crystalline HDI-650 and microphase separated three-component polyurethanes. This reflects the increased strain rate sensitivity in CoR of MDI-650 compared to MDIBDO-1000 described previously.

Dynamic stiffening from CoR and dynamic $\mathrm{T}_{\mathrm{g}}$ are correlated through the segmental dynamics of PTMO. Dynamic $T_{g}$ reflects the segmental mobility (reciprocal of segmental dynamics) of PTMO at a given temperature. We predict dynamic stiffening to be a result of a shift from rubbery to leathery or even glassy-like behavior as strain rates approach the segmental mobility of PTMO. Additionally, this shift would impede dissociation of the intermolecular hydrogen bonds. While these materials will all 


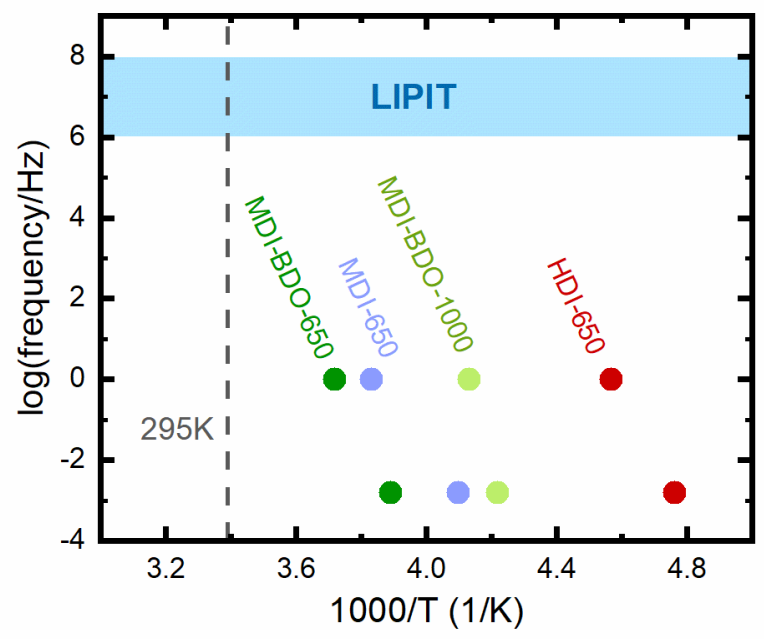

Figure 4. Comparison of the shift of $\mathrm{T}_{\mathrm{g}}$ obtained from DMA $(1 \mathrm{~Hz})$ and calorimetric $\mathrm{T}_{\mathrm{g}}$ from DSC (equivalent to $0.0016 \mathrm{~Hz}$ ) for polyurethanes. The vertical dotted line marks room temperature and the blue zone represents strain rates associated with LIPIT impacts. Overall, $T_{g}$ Notably, the $T_{g}$ of MDI-650 displays greatest sensitivity to frequency, reflecting the greater sensitivity to strain rate observed in its CoR.

presumably undergo room temperature glass transition at a sufficiently high strain rate, MDI-BDO-650 is the most likely to do so at the strain rates achievable with the LIPIT. The exact strain rate required for such a transition can be determined with broadband dielectric relaxation spectroscopy.

We investigate segmental dynamics at the molecular level via ${ }^{13} \mathrm{C}$ ssNMR dipolar dephasing measurements, specifically the peak at $\sim 71 \mathrm{ppm}$ corresponding to the ether carbon of PTMO. In brief, the dipolar dephasing was determined based on the evolution of the peak area while under the influence of ${ }^{1} \mathrm{H}^{-13} \mathrm{C}$ dipolar coupling. Fig. 5 compares the variation in peak area decay over time. More details into acquisition and analysis of ssNMR data is available in supplementary material. We fit the dipolar dephasing data with a two-component system, with each component representing a phase in the sample, to extract the respective decay time constants $\left(\mathrm{T}_{\mathrm{dd}}\right)$ and fractions, as reported in Table $\mathrm{S} 2$ (see supplementary material). Phases with higher mobility are usually better represented by an exponential decay, while glassy or crystalline phases by a Gaussian curve. Fast dephasing decay corresponds to the rigid phase and slow decay to the mobile phase. Decay time constants of the mobile phase $\left(\mathrm{T}_{\mathrm{dd}} \mathrm{m}\right)$ are listed for each polyurethane in Fig. 5, where lower $\mathrm{T}_{\mathrm{dd}}{ }^{\mathrm{m}}$ indicates lower segmental mobility of mobile phase PTMO. In microphase separated elastomers such as MDI-BDO-650, the presence of phase mixing or hard segments dissolved in the soft phase increases opportunity for intermolecular hydrogen bonding. We hypothesize that increased extent of intermolecular hydrogen bonding leads to lower segmental mobility and correspondingly lower $T_{d d}{ }^{m}$. The ordering of $T_{d d}{ }^{m}$ is consistent with that of both CoR and threshold velocity, especially for CoR where MDI-650 and MDI-BDO-1000 exhibit similar CoR and $T_{d d}{ }^{m}$. These findings support our understanding of dynamic stiffening and strengthening as result of a shift from rubbery to leathery or even glassy-like behavior as strain rates approach the segmental mobility of PTMO. 


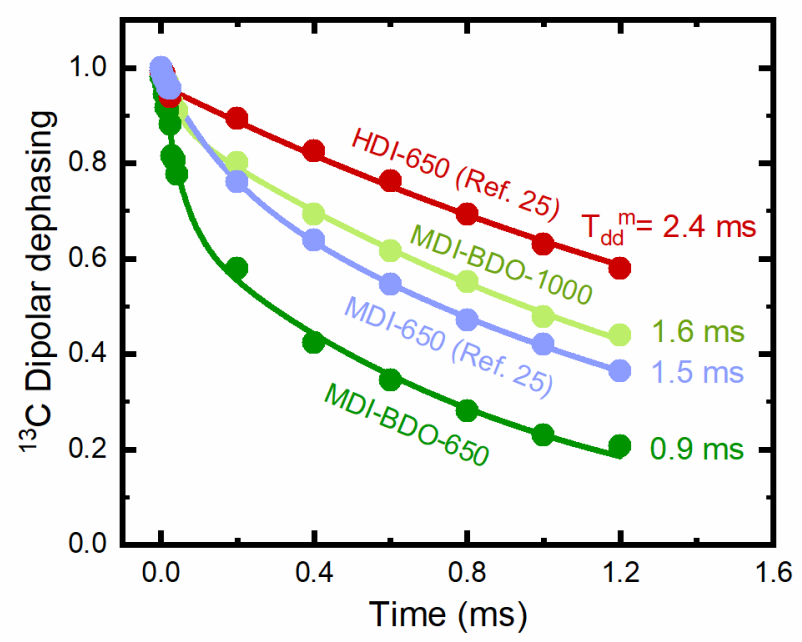

Figure 5. Comparison of ${ }^{13} \mathrm{C}$ ssNMR dipolar dephasing for polyurethanes. Lines are fits of the data to a two-phase system, mobile and rigid. Fractions and decay time constants are extracted for both phases. Decay time constants of the mobile phase $\left(\mathrm{T}_{\mathrm{dd}}{ }^{\mathrm{m}}\right)$ are reported here. For all fractions and constants, see Table S1 (see supplementary material). Data for MDI-650 and HDI-650 are reproduced from $\mathrm{Wu}$ et al. ${ }^{25}$ with permission from Elsevier.

Figure 6 displays a strong correlation between rigid-phase fraction of PTMO and threshold velocity. Notably, MDI-BDO-1000 and MDI-650 have quite different rigid-phase fractions despite having similar CoR curves. Moreover, the threshold velocities of these two materials are reflected in their rigid-phase fractions but not simply their hard segment contents. While the addition of BDO directly increases calculated hard segment content, it does not necessitate change in rigid-phase fraction of PTMO. This results from differences in crystallinity and microphase separation, revealed by comparison of HDI-650 to MDI-650 and MDI-BDO-1000 to MDI-650, respectively. Meanwhile, it is noteworthy that MDI-BDO-650 has a much higher rigid-phase fraction than MDI-BDO-1000. MDIBDO-650 has more carbamate moieties per unit chain length and fewer ether oxygen repeat units per respective PTMO segment. This provides MDI-BDO-650 with greater propensity for intermolecular hydrogen bonding and subsequently higher rigid-phase fraction compared to MDI-BDO-1000. These data establish a connection between rigid-phase fraction associated with PTMO and dynamic strengthening through intermolecular hydrogen bonding. 


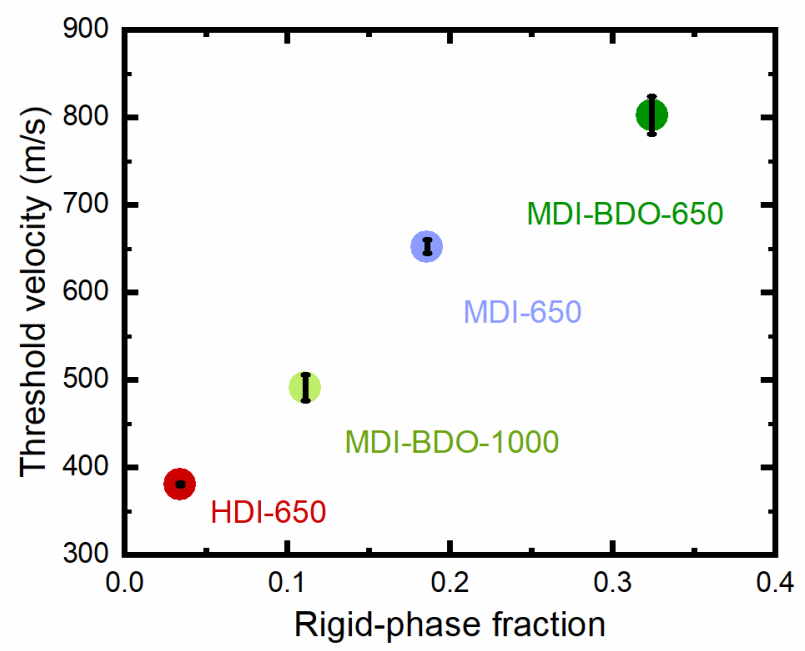

Figure 6. Comparison of threshold velocity from LIPIT to rigid-phase fraction of PTMO. Error bars for threshold velocity are shown. A strong correlation between the two values is observed, suggesting a rigid-phase fraction to play an especially important role in dynamic strengthening.

Lastly, we compare the impact response of MDI-BDO-650 with two polyureas, PU-650 and PU1000, shown in Fig. 7. Similar to polyurethane, these polyurea materials also show that the material with a shorter soft segment length has a higher CoR and threshold velocity than its counterpart. MDI-BDO650 and PU-1000 show remarkably similar CoR curves and nearly identical threshold velocities. It is further noteworthy that PU-650 exhibits higher CoR than MDI-BDO-650 with no observed threshold velocity at impacts up to $1300 \mathrm{~m} / \mathrm{s}$, despite the two materials having similar hard segment content. This dramatic difference in impact response likely results from differences in intersegmental hydrogen bonding between the two materials. Compared to monodentate hydrogen bonding in polyurethane, bidentate hydrogen bonding in polyurea yields stronger and more extensive hydrogen bonding interactions between the hard and soft segments, resulting in greater dynamic stiffening and dynamic strengthening. 


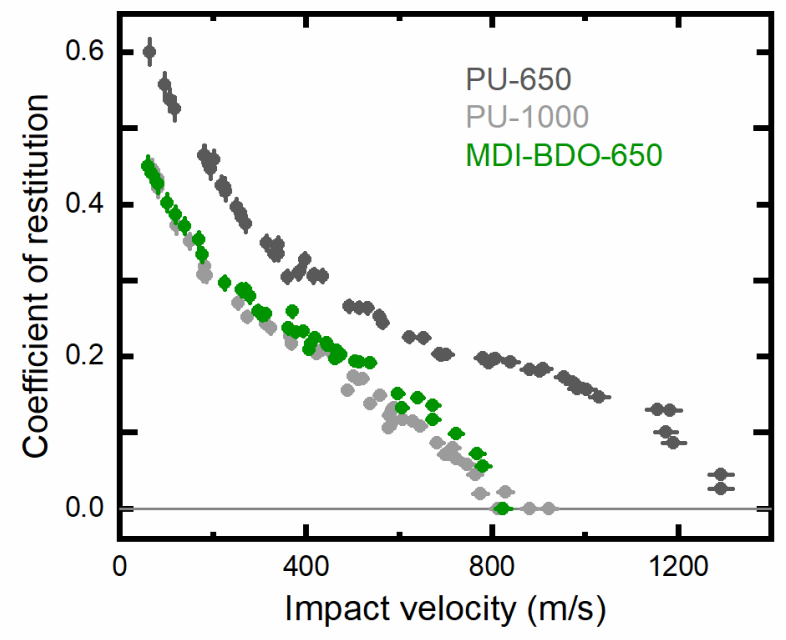

Figure 7. Comparison of coefficient of restitution over impact velocity for polyureas and MDIBDO-650 upon impact by $7.38 \mu \mathrm{m}$ silica spheres. Error bars are shown. Although PU-650 and MDI-BDO-650 have similar hard segment contents, the polyurea exhibits higher CoR and no observed threshold velocity with impacts up to $1300 \mathrm{~m} / \mathrm{s}$. These results suggest both the strength and extent of intersegmental hydrogen bonding to play important roles in material response.

In summary, we investigated the molecular dependencies of dynamic stiffening and strengthening in polyurethanes and polyureas by comparing high strain rate impact responses from the LIPIT to measurements from DSC, DMA, and ssNMR. Results show dynamic $\mathrm{T}_{\mathrm{g}}$ to be a useful predictor of high strain rate behaviors. We show that dynamic stiffening and dynamic strengthening depend strongly on segmental mobility of mobile-phase PTMO and that dynamic strengthening additionally depends on fraction of rigid-phase PTMO. The comparison of polyurethane and polyurea demonstrates that both soft segment length and hydrogen bonding type strongly influences impact response. The determinants of dynamic stiffening and strengthening identified in this study provide insight into the optimization of elastomeric materials. The LIPIT is used to establish a strategy for the systematic design and testing of high strain rate response in synthetic elastomers. 


\section{Acknowledgments}

This material is based upon work supported in part by the U. S. Army Research Office and CCDC Army Research Laboratory through the Institute for Soldier Nanotechnologies, under Cooperative Agreement Number W911NF-18-2-0048.

\section{Supplementary Material}

See supplementary material for materials characterization data obtained by DSC, as well as additional details into acquisition and analysis of phase fractions and dephasing decay time constants extracted from ${ }^{13} \mathrm{C}$ ssNMR data and calculated hard segment contents for polyurethanes.

\section{References}

${ }^{1}$ K. Kojio, S. Nakashima, and M. Furukawa, Polymer 48, 997 (2007).

${ }^{2}$ C.B. Wang and S.L. Cooper, Macromolecules 16, 775 (1983).

${ }^{3}$ J.P. Sheth, A. Aneja, G.L. Wilkes, E. Yilgor, G.E. Atilla, I. Yilgor, and F.L. Beyer, Polymer 45, 6919 (2004).

${ }^{4}$ E. Yilgor, M. Isik, and I. Yilgor, Macromolecules 43, 8588 (2010).

${ }^{5}$ R.B. Bogoslovov, C.M. Roland, and R.M. Gamache, Appl. Phys. Lett. 90, (2007).

${ }^{6}$ C.M. Roland, D. Fragiadakis, and R.M. Gamache, Compos. Struct. 92, 1059 (2010).

${ }^{7}$ D. Fragiadakis, R. Gamache, R.B. Bogoslovov, and C.M. Roland, Polymer 51, 178 (2010).

${ }^{8}$ D. Fragiadakis and J. Runt, Macromolecules 46, 4184 (2013).

${ }^{9}$ A.M. Castagna, D. Fragiadakis, H. Lee, T. Choi, and J. Runt, Macromolecules 44, 7831 (2011).

${ }^{10}$ C.M. Roland and R. Casalini, Polymer 48, 5747 (2007).

${ }^{11}$ K.E. Nugent, D.L. Ho, M.K. Vukmir, J.A. Pathak, T.H. Epps, J.N. Twigg, E.K. Lin, C.G. Robertson, P.H. Mott, and C.M. Roland, Macromolecules 41, 7543 (2008).

12 A.M. Castagna, A. Pangon, T. Choi, G.P. Dillon, and J. Runt, Macromolecules 45, 8438 (2012).

${ }^{13}$ S.S. Sarva and A.J. Hsieh, Polymer 50, 3007 (2009).

${ }^{14}$ R.G. Rinaldi, A.J. Hsieh, and M.C. Boyce, J. Polym. Sci. Part B Polym. Phys. 49, 123 (2011).

${ }^{15}$ K.E. Strawhecker, A.J. Hsieh, T.L. Chantawansri, Z.I. Kalcioglu, and K.J. Van Vliet, Polymer 54, 901 (2013).

${ }^{16}$ A.J. Hsieh, T.L. Chantawansri, W. Hu, K.E. Strawhecker, D.T. Casem, J.K. Eliason, K.A. Nelson, and E.M. Parsons, Polymer 55, 1883 (2014).

${ }^{17}$ W. Hu, N. V. Patil, and A.J. Hsieh, Polymer 100, 149 (2016).

${ }^{18}$ A.J. Hsieh, T.L. Chantawansri, W. Hu, J. Cain, and J.H. Yu, Polymer 95, 52 (2016).

${ }^{19}$ D. Veysset, A.J. Hsieh, S. Kooi, A.A. Maznev, K.A. Masser, and K.A. Nelson, Sci. Rep. 6, 1 (2016).

${ }^{20}$ D. Veysset, A.J. Hsieh, S.E. Kooi, and K.A. Nelson, Polymer 123, 30 (2017).

${ }^{21}$ A.J. Hsieh, D. Veysset, D.F. Miranda, S.E. Kooi, J. Runt, and K.A. Nelson, Polymer 146, 222 (2018).

${ }^{22}$ N.K. Bourne, J. Dyn. Behav. Mater. 2, 33 (2016).

${ }^{23}$ J.-H. Lee, D. Veysset, J.P. Singer, M. Retsch, G. Saini, T. Pezeril, K.A. Nelson, and E.L. Thomas, Nat. Commun. 3, 1164 (2012).

${ }^{24}$ D. Veysset, S.E. Kooi, A.A. Maznev, S. Tang, A.S. Mijailovic, Y.J. Yang, K. Geiser, K.J. Van Vliet, B.D. Olsen, K.A. Nelson, J. of the Mech. Beh. of Biomed. Mat. 86, 71-76 (2018).

${ }^{25}$ Y.C.M. Wu, W. Hu, Y. Sun, D. Veysset, S.E. Kooi, K.A. Nelson, T.M. Swager, and A.J. Hsieh, Polymer 168, 218 (2019).

${ }^{26}$ J. Lee, P.E. Loya, J. Lou, and E.L. Thomas, Science 346, 1092 (2014).

${ }^{27}$ R. Thevamaran, O. Lawal, S. Yazdi, S.J. Jeon, J.H. Lee, and E.L. Thomas, Science 354, 312 (2016).

${ }^{28}$ M. Hassani-Gangaraj, D. Veysset, K.A. Nelson, and C.A. Schuh, Scr. Mater. 145, 9 (2018). 


\section{Supplementary materials}

S1. Material characteristics

Differential scanning calorimetry (DSC) data were obtained with a TA Instruments Discovery DSC instrument, using a heat/cool/heat cycle from -90 to $180{ }^{\circ} \mathrm{C}$ at a heating/cooling rate of $10{ }^{\circ} \mathrm{C} / \mathrm{min}$, under a nitrogen atmosphere. The trend in $\mathrm{T}_{\mathrm{g}}$ is as follows: MDI-BDO-650 (257K) > MDI-BDO-1000 $(244 \mathrm{~K})>\mathrm{MDI}-650(237 \mathrm{~K})>\mathrm{HDI}-650(210 \mathrm{~K})$. Table S1 lists the $\mathrm{T}_{\mathrm{g}}$ data obtained from DSC as well as from DMA for these polyurethanes that are shown in the dynamic $\mathrm{T}_{\mathrm{g}}$ plot in Fig. 4.

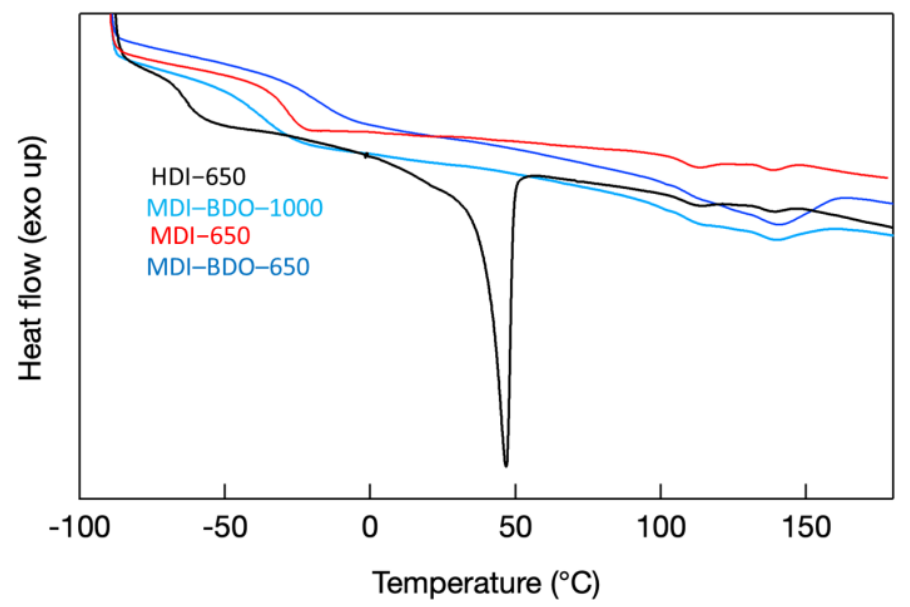

Fig. S1. DSC thermograms of HDI-650 (black), MDI-BDO-1000 (light blue), MDI-650 (red), and MDIBDO-650 (blue).

Table S1. $\mathrm{T}_{\mathrm{g}}$ s obtained from DSC and DMA for the tested polyurethanes.

\begin{tabular}{|c|c|c|}
\cline { 2 - 3 } \multicolumn{1}{c|}{} & $\mathbf{T}_{\mathbf{g}}(\mathbf{K}), \mathbf{D S C}$ & $\mathbf{T}_{\mathbf{g}}(\mathbf{K}), \mathbf{D M A}$ \\
\hline HDI-650 & 210 & 219 \\
\hline MDI-BDO-1000 & 237 & 242 \\
\hline MDI-650 & 244 & 261 \\
\hline MDI-BDO-650 & 257 & 269 \\
\hline
\end{tabular}

\section{S. $2{ }^{13} \mathrm{C}$ ssNMR dipolar dephasing characterization}

In brief, the dipolar dephasing was determined based on the evolution of the peak area at $\sim 71$ ppm, corresponding to the ether carbon of PTMO, under the influence of ${ }^{1} \mathrm{H}-{ }^{13} \mathrm{C}$ dipolar coupling. Note that these results are obtained for ether carbons of PTMO that are $>=3-4$ bonds away from the carbamate groups. Meanwhile, rigid PTMO segments have strong ${ }^{1} \mathrm{H}^{-13} \mathrm{C}$ dipolar coupling leading to faster signal decay, whereas the ${ }^{1} \mathrm{H}_{-}{ }^{13} \mathrm{C}$ dipolar coupling strength of the mobile PTMO segments is attenuated by faster molecular dynamics, resulting in slower decay. The evolution times were between 1 and $42 \mu \mathrm{s}$, as well as multiples of rotor echo ${ }^{17}$, which capture the fast-decaying and slow-decaying component, respectively, while minimizing the modulating effect of MAS on ${ }^{1} \mathrm{H}-{ }^{13} \mathrm{C}$ dipolar coupling. Additional experimental details of the ${ }^{13} \mathrm{C}$ dipolar dephasing measurements can be found in ref. 17 . In 
Fig. S2, representative dipolar dephasing spectra highlighting the PTMO ether carbon signal at $~ 71$ ppm of MDI-PTMO-650 obtained at evolution time of $1 \mu \mathrm{s}, 36 \mu \mathrm{s}$, and $1.2 \mathrm{~ms}$ are shown. Further, we fit the dipolar dephasing decay data with a two-component model with each component representing a phase in the sample. Phases with higher mobility are usually better represented by an exponential decay, while glassy or crystalline phases often well-fit with a Gaussian curve. Table S2 lists the fitting models, the phase fractions and dephasing decay time constants obtained for the respected rigid and mobile phases for polyurethanes along with corresponding hard segment content.

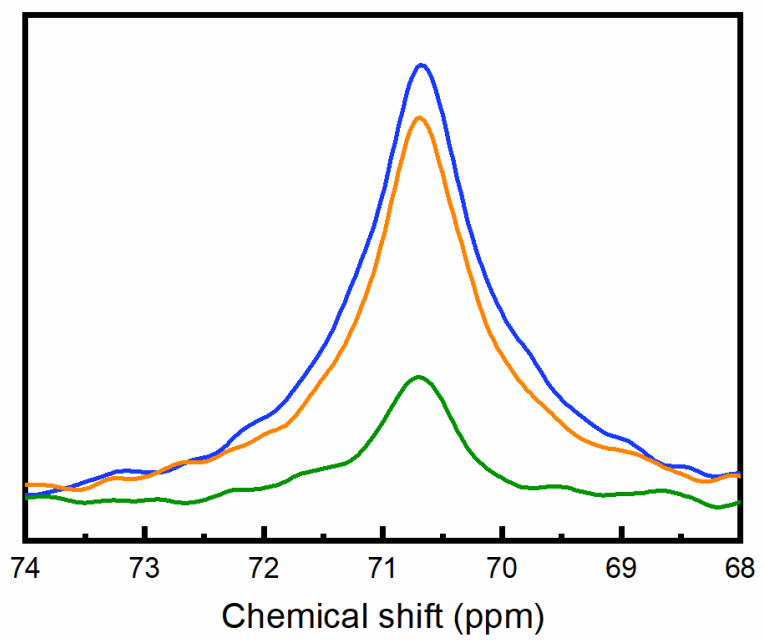

Fig. S2. Dipolar dephasing spectra of MDI-BDO-650 at evolution time of $1 \mu$ s (blue), $36 \mu s$ (orange), and $1.2 \mathrm{~ms}$ (green), highlighting the PTMO ether carbon signal at $\sim 71 \mathrm{ppm}$.

Table S2. Dipolar dephasing data from ${ }^{13} \mathrm{C}$ ssNMR of polyurethanes are fitted to a two-component system using the stated fitting models. Phase fractions and dephasing decay time constants are extracted from the fits for rigid and mobile phases along with hard segment.

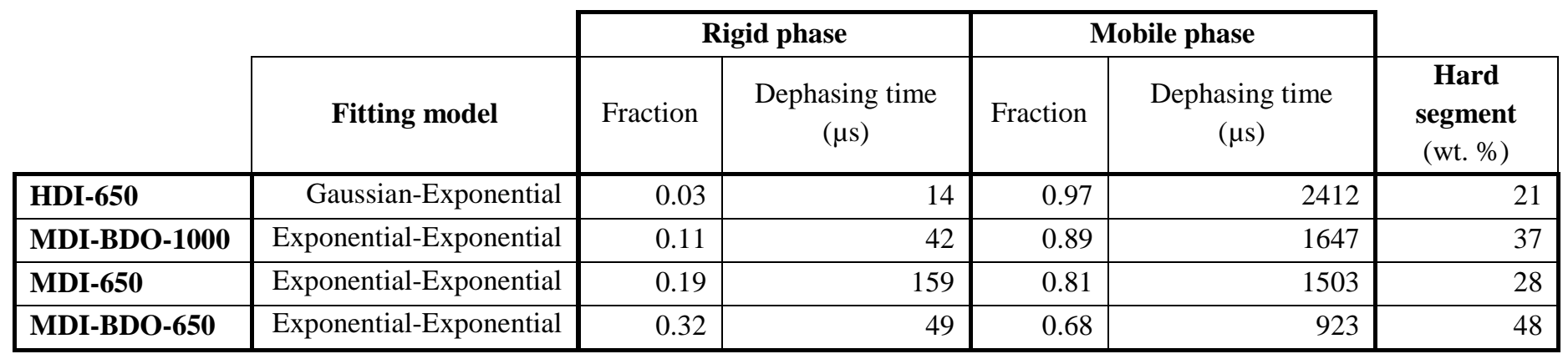

